\title{
Uso de Jogos Digitais Educativos na Educação Profissional e Tecnológica fundamentados em Teorias de Aprendizagem
}

\section{Use of Educational Digital Games in Professional and Technological Education based on Learning Theories}

\author{
ALTAIR FÁBIO SILVÉRIO RIBEIRO \\ ProfEPT/Instituto Federal Goiano \\ ROBERTA MARTINS MENDONÇA GOMES \\ ProfEPT/Instituto Federal Goiano \\ JOSÉ ROBERTO CRUZ E SILVA \\ ProfEPT/Instituto Federal Goiano \\ JAINER DIOGO VIEIRA MATOS \\ ProfEPT/Instituto Federal Goiano \\ JÚLIO CÉSAR FERREIRA \\ ProfEPT/Instituto Federal Goiano \\ FERNANDO BARBOSA MATOS \\ ProfEPT/Instituto Federal Goiano
}

\begin{abstract}
Resumo: Estudos voltados a recursos que interferem diretamente no campo educacional tornam-se cada vez mais pertinentes na era digital, uma vez que, é indispensável o uso de ferramentas que favoreçam os processos de ensino e aprendizagem. Nessa perspectiva surgem os jogos digitais educativos (JDE), cuja utilização crescente como ferramenta educacional traz também a necessidade de reflexões, em especial sobre as teorias de aprendizagem que estão embasando práticas educativas por meio desses recursos. Assim, por meio de uma revisão sistemática, buscou-se como objetivo geral verificar qual a teoria de aprendizagem é mais citada em artigos encontrados na base de periódicos da Capes, publicados de 2014 a 2018, para fundamentar a utilização de JDE na Educação Profissional e Tecnológica (EPT). A pesquisa realizada traz indícios que sugerem a ocorrência de um número reduzido de artigos sobre JDE em disciplinas técnicas da EPT e que inexiste prevalência de uma teoria de aprendizagem fundamentando JDE.
\end{abstract}

Palavras-chave: Jogos Digitais Educativos. Jogos Eletrônicos Didáticos. Teorias de Aprendizagem. Educação Profissional e Tecnológica. Capes.

Abstract: Studies focused on resources that directly interfere in the educational field become increasingly relevant in the digital age, since it is indispensable to use tools that favor the teaching and learning processes. In this perspective the digital educational games (DEG) 
emerge, whose increasing use as an educational tool also brings the need for reflections, especially on the learning theories that are supporting educational practices through these resources. Thus, means of a systematic review, sought as general objective to verify which learning theory is most cited in articles found in the periodicals of Capes, published from 2015 to 2018, to support the use of DEG in Vocational and Technological Education (VTE). The research suggests that there is a small number of DEG articles in technical disciplines of VTE in and that there is no prevalence of a theory of learning give basis those DEG.

Keywords: Digital Educational Games. Electronic Educational Games. Learning Theory. Professional and Technological Education. Capes. 


\section{Introdução}

Os avanços tecnológicos e a rapidez com que as inovações se integram à sociedade trazem pertinência aos estudos voltados a recursos e ferramentas que interferem diretamente nas atividades dos diversos setores, incluindo o campo educacional. Na era digital, o uso de ferramentas para auxiliar processos de ensino e aprendizagem deixa de ser um diferencial para se tornar um elemento-chave capaz de fazer pessoas pertencerem, de fato, à sociedade da informação em rede, ou cibercultura (Lévy, 1999), na qual todos os indivíduos estão conectados.

Observando o grande número de recursos tecnológicos e sabendo a importância de aliar educação e tecnologia na promoção de um ensino atraente, serão destacados neste artigo os Jogos Digitais Educativos (JDE). Neste contexto, surge o seguinte questionamento: qual teoria de aprendizagem é mais referenciada em artigos científicos levantados a partir de pesquisa na base de dados de periódicos da Coordenação de Aperfeiçoamento de Pessoal de Nível Superior (Capes), de 2014 a 2018, para fundamentar a utilização de JDE em disciplinas técnicas na Educação Profissional e Tecnológica (EPT)?

Como hipótese de resposta, os autores do presente estudo suspeitam que existem poucos artigos indexados na base de periódicos da Capes, abordando a utilização de JDE em EPT e menor ainda o uso de teorias de aprendizagem para embasamento de trabalhos com essa temática. Dentro desse cenário propõe-se um estudo que tem como objetivo geral verificar qual a teoria de aprendizagem é mais citada em artigos encontrados em pesquisa via base de periódicos da Capes, dos anos de 2014 a 2018, para fundamentar a utilização de JDE na EPT.

Neste estudo, os seguintes objetivos específicos também serão buscados: verificar qual a terminologia mais usada nos títulos de artigos científicos para fazer referência a JDE e identificar o número de artigos no portal Capes, que tratam especificamente de aplicação de JDE em disciplinas técnicas da EPT.

Visando atender aos objetivos estabelecidos, este estudo fará uso de uma pesquisa de natureza descritiva fazendo uso de revisão sistemática da literatura. Pesquisas similares a este trabalho, porém não aplicada à modalidade EPT, encontram-se destacadas em alguns estudos, como os de Hwang e Wu (2012) e de Ribeiro et al. (2015).

Entende-se que a proposta de estudo se justifica uma vez que, qualquer ferramenta utilizada como mediadora na prática educativa necessita de uma concepção pedagógica que atenda aos objetivos e resultados esperados para uma sólida construção do conhecimento. Tal fundamento é encontrado em teorias de aprendizagem. O autor Moreira (2017, p.12) afirma que "Uma teoria de aprendizagem é, então, uma construção humana para interpretar sistematicamente a área de conhecimento que chamamos de aprendizagem".

Reconhece-se a importância em descobrir quais são as teorias de aprendizagem mais utilizadas na literatura científica para fundamentar o desenvolvimento e aplicação de JDE. A partir desse conhecimento é possível ampliar a discussão sobre a prevalência de determinada teoria e em quais âmbitos são mais exploradas. 
Assim pode ser estabelecida uma temática para a elaboração de estudos sobre o estado da arte referente a trabalhos realizados por pesquisadores que se debruçam nas análises sobre os JDE na modalidade de ensino em EPT especificamente em se tratando de disciplinas técnicas. Lourenço (2012) destaca a importância de pesquisa sobre JDE (games), afirmando que:

\footnotetext{
Desta forma, o levantamento das pesquisas sobre games entendidos como meios e mediações comunicacionais em inter-relação com a educação visa a possibilitar um entendimento do estado da arte das investigações científicas sobre este objeto, o game, vislumbrando os caminhos e as tendências das pesquisas nessa área. (LOURENÇO, 2012, p. 14).
}

Com o intuito de facilitar a possibilidade de replicação desse estudo por outros pesquisadores, duas etapas foram definidas, sendo uma mais geral e outra mais restrita. Ambas são detalhadas nos procedimentos metodológicos. Na Etapa 1 utiliza-se uma combinação de filtros de pesquisa para permitir resultados mais abrangentes, enquanto na Etapa 2 é buscado o refinamento do trabalho, ou seja, encontrar artigos específicos que atendam aos requisitos estabelecidos nos objetivos desse artigo.

\section{Fundamentação Teórica}

Para subsidiar o presente estudo, serão abordados os seguintes temas: Jogos Digitais Educativos, Teorias de Aprendizagem, Educação Profissional e Tecnológica e o portal Capes como locus da pesquisa. Nesse sentido, cada assunto será apresentado de forma resumida, buscando suas correlações, dentro do contexto da pesquisa proposta.

\subsection{Jogos Digitais Educativos (JDE)}

Huizinga (1990) analisa o jogo como um fator distinto e fundamental presente em tudo que acontece no mundo, defendendo que o jogo para o ser humano é tão importante quanto o raciocínio e a fabricação de objetos e, dessa forma, propõe que a expressão Homo ludens, mereça um lugar na nossa nomenclatura juntamente com Homo sapiens e Homo faber. Destaca-se que em seus trabalhos, Avedon e Sutton (1971), Caillois (1990) e Huizinga (1990) defendem a ideia do jogo como atividade voluntária e de interação lúdica. Segundo esses estudiosos, caso exista o caráter obrigatório para participação, o jogo, perderia de uma só vez sua qualidade atrativa e alegre como diversão. Conforme Lima (2008, p. 22), "a partir do início do século XIX, estudiosos assumiram o jogo como objeto de investigação científica e elaboraram pesquisas que procuraram compreender e explicar a importância desse tipo de atividade, na vida dos homens e dos animais".

Posteriormente, e também em função do desenvolvimento das tecnologias digitais, novos estudos foram observando a importância dos jogos eletrônicos (aqui considerado como uma das possíveis nomenclaturas de JDE encontradas na literatura científica) e sua relação direta com a sociedade, bem como seu potencial educacional. Assim, autores como Prensky (2006, 2008), Lim (2008), Vos et al. (2011) e Yang e Chang (2013) apresentaram estudos que destacam o potencial dos jogos digitais como uma das abordagens inovadoras mais promissoras para a educação de crianças e adolescentes, uma vez que esses jogos estão entre 
os passatempos preferidos nessas faixas etárias, despertando a motivação intrínseca, tão almejada por educadores (Falcão; Falcão; Hazin, 2016).

Conforme Alves (2008), as investigações sobre a temática de JDE têm início em meados da década de 1980. A partir desse período, os investigadores da Europa e Estados Unidos começam a divulgar resultados de pesquisas relacionando jogos eletrônicos e aprendizagem.

Observa-se que a definição do termo jogo apresentada não é um consenso, mesmo porque a palavra "jogo" apresenta significados diferentes de acordo com o contexto. Como afirma Brougère (2003), o caráter polissêmico do termo deriva de culturas com seus modos próprios e assim, a noção de jogo provém da sua compreensão em diferentes contextos sociais, mas sendo considerado como um fato social. Segundo o autor, mesmo Piaget não chega a estabelecer um conceito de jogo.

Ainda nessa perspectiva, Lima (2008) destaca que o termo jogo não é abstrato e que, vários autores estabeleceram suas próprias análises de acordo com o contexto em que seus estudos foram desenvolvidos, afirmando assim que "não há um único conceito científico estabelecido e definitivo sobre o vocábulo" (LIMA, 2008, p.36).

Mesmo sem uma exata e consensual definição, a utilização crescente dos jogos em seu formato digital como ferramenta educacional traz também a necessidade de reflexão sobre a forma com que os JDE estão sendo desenvolvidos, bem como quais são as teorias de aprendizagem que estão embasando o processo de ensino e aprendizagem por meio desses recursos. Essa reflexão é necessária, uma vez que todo e qualquer recurso que não tenha uma intencionalidade e objetivos claros acaba perdendo seu foco e, consequentemente, pode não alcançar ou alcançar parcialmente seu objetivo.

\subsection{Teorias de aprendizagem}

Conforme Moreira (2008), uma teoria de aprendizagem representa o ponto de vista de um autor/pesquisador que tenta explicar o que é aprendizagem e porque funciona de tal forma. As teorias de aprendizagem podem ser subdivididas em três filosofias subjacentes, a saber: comportamentalista (behaviorismo), cognitivista (construtivismo) e a humanista. Observa-se, contudo, que nem sempre é possível enquadrar determinada teoria de aprendizagem em apenas uma corrente filosófica.

A abordagem comportamentalista (behaviorista) relaciona-se com os comportamentos mensuráveis do sujeito nas respostas aos estímulos externos, entendendo assim que o comportamento é controlado pelas consequências. Na aprendizagem, os objetivos comportamentais definiam aquilo que o aluno deveria ser capaz de aprender e em quais condições. Sendo os objetivos atingidos, entendia-se que havia ocorrido aprendizagem (MOREIRA, 2008).

O cognitivismo enfatiza a cognição, ou seja, os processos mentais dos quais decorrem o conhecimento, se ocupando da atribuição de significados, da compreensão ao uso da informação envolvida na atividade mental. Nessa perspectiva de que a cognição ocorre por construção chega-se ao construtivismo. Observa-se que não há um método e sim teorias 
construtivistas que, no ensino, apresenta o aluno como um agente da sua estrutura cognitiva. (MOREIRA, 2008).

O humanismo, por sua vez, percebe o aprendiz na sua totalidade, não sendo possível falar do comportamento ou da cognição sem considerar o domínio afetivo e os sentimentos das pessoas. Nessa perspectiva, o educando é visto como um ser que pensa, sente e age de maneira integrada (MOREIRA, 2008). Além das teorias de aprendizagem descritas acima, aponta-se a Teoria de Aprendizagem Experimental e a Taxonomia de Bloom.

Como teoria da aprendizagem com foco no desenvolvimento do ser adulto, em especial o profissional, conforme Pimentel (2007), surge a teoria da aprendizagem experiencial (teoria kolbiana), fundada pelo americano David Kolb, com uma perspectiva holística e integrativa aproximando-a das tendências voltadas ao pensamento reflexivo para melhoria da qualificação profissional, sendo a experimentação fator essencial para estabelecer relação entre teoria e prática. Essa teoria afirma que a origem do desenvolvimento profissional se encontra no processo de aprendizagem, aludindo a princípios e conceitos defendidos por Vygotsky (1998).

Já a Taxonomia de Bloom et al. (1972) teve como intuito a organização classificatória que permitiu observar que a maior proporção de objetivos educativos recaia sobre três domínios: o afetivo, o cognitivo e o psicomotor, observando que, nenhum objetivo estaria em uma classe, sendo totalmente destituído das demais. Dessa forma, a teoria tem por objetivo favorecer a comunicação entre professores e professores pesquisadores do currículo, ajudando assim na conscientização com relação a técnicas disponíveis para avaliação do desenvolvimento dos estudantes.

\subsection{Educação Profissional e Tecnológica (EPT)}

A Educação Profissional e Tecnológica, teve origem no Brasil, com a formação profissionalizante das Escolas de Aprendizes Artífices durante o governo de Nilo Peçanha em 1909, mas apenas em 1941, com a Reforma Capanema, é que o ensino profissional passou a contemplar a formação de nível médio (MOURA, 2007). Observando à legislação brasileira, sabe-se que, apenas após a Lei de Diretrizes e Bases da Educação Nacional - LDB n. 9394/96 é que a educação profissional passou a ser considerada uma das etapas da educação básica, vinculando a educação escolar ao mundo do trabalho e à prática social. Nos últimos anos, a educação profissional passou por mudanças, principalmente aquelas relacionadas à formação integrada com garantia ao educando do direito a uma formação omnilateral para a leitura do mundo e para a atuação como cidadão pertencente a um país, ou seja, um ser integrado na sua totalidade ao mundo que o cerca (FRIGOTTO; CIAVATTA, 2012).

Ressalta-se que, dentro da educação profissional existem duas definições de disciplinas, as propedêuticas e as técnicas. Para melhor entendimento, define-se disciplina técnica como aquela de caráter profissionalizante que compõe o currículo de cursos da EPT de nível médio. Já as disciplinas propedêuticas, são aquelas de formação geral que compõem a Base Nacional Comum Curricular, tais como: matemática, português, história, geografia, química, entre outras. 


\subsection{Base de Dados de Periódicos da Coordenação de Aperfeiçoamento de Pessoal de Nível Superior (Capes)}

A plataforma Capes é a iniciativa do gênero com a maior capilaridade no planeta, cobrindo todo o território nacional (BRASIL, 2000a). Sendo, portanto, uma ferramenta fundamental de fomento, avaliação e regulação dos cursos de pós-graduação e desenvolvimento da pesquisa científica no Brasil. A escolha do portal Capes como locus da pesquisa se deu pelas razões expostas na sequência.

A citada plataforma é considerado um modelo de consórcio de bibliotecas único no mundo, pois é inteiramente financiado pelo governo brasileiro, oferecendo acesso a textos completos disponíveis em mais de 38 mil publicações periódicas (internacionais e nacionais) e a diversas bases de dados que reúnem desde referências e resumos de trabalhos acadêmicos e científicos até normas técnicas, patentes, teses e dissertações, cobrindo todas as áreas do conhecimento (BRASIL, 2000). Inclui também uma seleção de importantes fontes de informação científica e tecnológica de acesso gratuito na web.

Cabe destacar que existem outras plataformas para acesso às publicações periódicas científicas e acadêmicas tais como Scopus, Web of Science, Google Scholar entre outras. Porém, no presente estudo, os autores deliberadamente optaram pelo uso do portal Capes, objetivando também proporcionar um maior detalhamento no processo de busca que possa ser replicado especificamente nessa plataforma.

\section{Metodologia}

Nesta pesquisa foi utilizado o estudo de natureza descritiva fazendo uso de revisão sistemática da literatura. Como pode ser observado em Galvão e Pereira (2014, p. 183), revisão sistemática da literatura é um tipo de investigação focada em questão bem definida, que visa identificar, selecionar, avaliar e sintetizar as evidências relevantes disponíveis.

Para a consecução da revisão sistemática, a pesquisa foi delimitada em duas etapas. Na sequência cada uma delas é descrita em detalhes, de forma a permitir sua replicação. Destacase que os dados foram coletados no dia 25 de novembro de 2018.

\subsection{ETAPA 1: Definição dos filtros para a revisão sistemática na base de periódicos do portal Capes}

\subsubsection{Acesso ao portal Capes}

Os seguintes passos foram executados no site http://www.periodicos.capes.gov.br/: seleção em sequência dos campos - Busca; Buscar assunto e Busca avançada. Ao final dos passos descritos, a imagem da Figura 1 foi visualizada. 
Figura 1 - Janela de busca por assunto portal Capes.

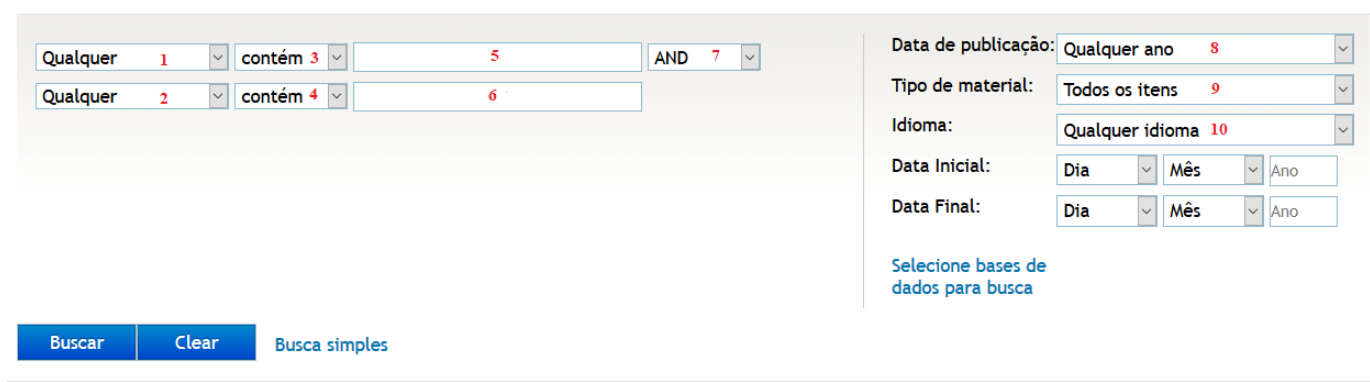

Fonte: Elaborada pelos autores.

\subsubsection{Palavras-chave (descritores ou unitermos) para pesquisa}

Como não há um padrão único de nomenclatura para referir-se a jogo digital no contexto educativo, diversos descritores foram utilizados, objetivando uma maior abrangência de terminologias. Destaca-se que para cada etapa de busca é inserida uma palavra chave no campo 5 e/ou campo 6. Esses campos podem ser vistos na Figura 1.

A seguir são elencados os descritores escolhidos: jogo digital, jogo eletrônico, jogo computacional, game educativo, game educacional, jogo sério, jogo and educação profissional, game and educação profissional, objeto de aprendizagem and educação profissional, serious game and vocational, game and vocational education, digital educational game.

\subsubsection{Critérios de exclusão}

Nos campos 1 e 2 existem as seguintes opções de filtros: qualquer, no título, como autor e no assunto. Já nos campos 3 e 4 as opções são: contém, é exato e começa com. 0 campo 7 permite as opções para pesquisas compostas por dois tipos de descritores, unidos por um dos operadores and, or e not.

Nessa primeira etapa de processo de busca, a configuração abaixo foi aplicada: Filtro Campo 1: qualquer; Filtro Campo 2: qualquer; Filtro Campo 3: é exato; Filtro Campo 4: é exato; Filtro Campo 7: and; Filtro Campo 8: últimos 5 anos; Filtro Campo 9: artigos; Filtro Campo 10: qualquer idioma e Periódicos revisados por pares. Ressalta-se que a escolha do intervalo de 5 anos como filtro, deveu-se ao fato de que se priorizou a busca por trabalhos mais atuais, tendo em vista que boa parte de estudos sobre a temática de jogos digitais voltados para o processo de ensino e aprendizagem, também são relativamente recentes, quando se leva em conta, toda a história da educação.

\subsection{ETAPA 2: Critérios de refinamento da pesquisa}

Após a execução do procedimento proposto na Etapa 1, foi estabelecida uma nova configuração para refinar a pesquisa, como segue: Filtro Campo 1: no título; Filtro Campo 2: qualquer; Filtro Campo 3: é exato; Filtro Campo 4: é exato; Filtro Campo 7: and; Filtro Campo 8: últimos 5 anos; Filtro Campo 9: artigos; Filtro Campo 10: qualquer idioma. 
Nessa etapa foram selecionados os artigos que tinham em seu título os descritores indicados. Como critério de seleção para o tipo de teoria de aprendizagem, foram consideradas apenas as teorias explicitamente indicadas neste estudo. Nos casos em que a teoria de aprendizagem não foi informada, mas que um ou mais teóricos pertencentes à determinada filosofia de aprendizagem foram citados no texto dos artigos fundamentando a questão pedagógica, esse critério também foi adotado.

Dando sequência à investigação, foi feita a leitura completa de cada artigo selecionado na Etapa 2 de acordo com os seguintes critérios preestabelecidos: artigos que se encontram disponibilizados gratuitamente na internet e que tratam especificamente de jogos digitais no contexto educativo.

\section{Resultados e discussão}

Após a execução dos procedimentos metodológicos, foi possível tratar os dados coletados e elaborar os quadros indicados na sequência. Destaca-se que, os quadros possuem as colunas: Número de Artigos Encontrados na Etapa 1 (NAE1); Número de Artigos Encontrados na Etapa 2 (NAE2); Detalhes dos Artigos Filtrados na Etapa 2; Artigos com tema de JDE na EPT para disciplinas técnicas (EPT-DT) e Teoria de Aprendizagem (TA).

Os seguintes termos com suas respectivas siglas foram utilizados nos quadros: Comportamentalismo (COM); Cognitivismo (COG); Humanismo (HUM); Taxonomia de Bloom (TB); Aprendizagem Experiencial (AE); Não Apresenta Teoria de Aprendizagem (NATA); Desconsiderado da Pesquisa (DP); Campo 5 (C5); Campo 6 (C6). O símbolo (*), indica que o artigo foi encontrado em mais de um critério de seleção. Nesta situação foram encontrados dois artigos repetidos.

O Quadro 1 indica os dados obtidos fazendo uso do seguinte critério de pesquisa - Critério 1: $\mathrm{C5}$ = jogo digital e C6 = vazio (nenhum termo digitado).

Quadro 1 - Artigos selecionados pelo Critério 1.

\begin{tabular}{|c|c|c|c|c|}
\hline NAE1 & NAE2 & DETALHES DOS ARTIGOS FILTRADOS NA ETAPA 2 & EPT-DT & TA \\
\hline \multirow{3}{*}{12} & \multirow{3}{*}{3} & $\begin{array}{l}\text { ARTIGO } 01 \text { - Título: A aprendizagem de métodos } \\
\text { numéricos com o jogo digital Handles in Scratch } 2.0 \\
\text { Autores: Marcos Henrique de Paula Dias Da Silva }\end{array}$ & 1 & NATA \\
\hline & & $\begin{array}{c}\text { ARTIGO } 02 \text { - Título: Crayon Sharks: um estudo de caso } \\
\text { sobre o design e aplicação de um jogo digital para o } \\
\text { ensino de ciências } \\
\text { Autores: Silva, M. ; Araujo, R. }\end{array}$ & 0 & HUM \\
\hline & & $\begin{array}{c}\text { ARTIGO } 03 \text { - Título: A utilização de tecnologias na } \\
\text { cartografia escolar: jogo digital para a alfabetização } \\
\text { cartográfica } \\
\text { Autores: Dambros, Gabriela; Marmentini Rovani, Franciele } \\
\text { Francisca; Henrique Quoos, João; Cassol, Roberto. }\end{array}$ & 0 & NATA \\
\hline
\end{tabular}

Fonte: Elaborado pelos autores.

O Quadro 2 indica os dados obtidos fazendo uso do seguinte critério de pesquisa - Critério 2: $\mathrm{C5}$ = jogo eletrônico e C6 = vazio (nenhum termo digitado) . 
Quadro 2 - Artigos selecionados pelo Critério 2.

\begin{tabular}{|c|c|c|c|c|}
\hline NAE1 & NAE2 & DETALHES DOS ARTIGOS FILTRADOS NA ETAPA 2 & $\begin{array}{c}\text { EPT- } \\
\text { DT }\end{array}$ & TA \\
\hline 7 & 1 & $\begin{array}{c}\text { ARTIGO 04 - Título: jogo eletrônico e educação musical: } \\
\text { limites e possibilidades }\end{array}$ & $\begin{array}{c}\text { Autores: Luciana Carolina Fernandes de Faria; Sheila } \\
\text { Regiane Franceschini; Beatriz de Oliveira Ogata; Karen } \\
\text { Rodrigues da Rocha; Wellington Ricardo de Souza. }\end{array}$ & 0 \\
HUM \\
\hline
\end{tabular}

Fonte: Elaborado pelos autores.

O Quadro 3 indica os dados obtidos fazendo uso do seguinte critério de pesquisa - Critério

3: $\mathrm{C} 5=$ jogo computacional e $\mathrm{C} 6$ = vazio (nenhum termo digitado).

Quadro 3 - Artigos selecionados pelo Critério 3.

\begin{tabular}{|c|c|c|c|c|}
\hline NAE1 & NAE2 & DETALHES DOS ARTIGOS FILTRADOS NA ETAPA 2 & EPT-DT & TA \\
\hline \multirow{3}{*}{3} & \multirow{3}{*}{3} & $\begin{array}{c}\text { ARTIGO } 05 \text { - Título: Jogo computacional e resolução de } \\
\text { problemas: três estudos de casos - Computer game and } \\
\text { problem solving: three case studies } \\
\text { Autores: Althaus, Neiva; Dullius, Maria. }\end{array}$ & 0 & NATA \\
\hline & & $\begin{array}{c}\text { ARTIGO } 06 \text { - Título: Modelo de dobramento de proteína } \\
\text { em jogo computacional } \\
\text { Autores: Renan Martinez Da Luz; Diana Francisca } \\
\text { Adamatti; Adriano Velasque Werhli. }\end{array}$ & 0 & NATA \\
\hline & & $\begin{array}{c}\text { ARTIGO } 07 \text { - Título: The Sims: Jogo Computacional como } \\
\text { uma Ferramenta Pedagógica na Construção do } \\
\text { Conhecimento Matemático } \\
\text { Autores: Rúbia Juliana Gomes Fernandes; Guataçara dos } \\
\text { Santos Junior. }\end{array}$ & 0 & COG \\
\hline
\end{tabular}

Fonte: Elaborado pelos autores.

O Quadro 4 indica os dados obtidos fazendo uso do seguinte critério de pesquisa - Critério 4: $\mathrm{C5}$ = jogo sério e C6 = vazio (nenhum termo digitado).

Quadro 4 - Artigos selecionados pelo Critério 4.

\begin{tabular}{|c|c|c|c|c|}
\hline NAE1 & NAE2 & DETALHES DOS ARTIGOS FILTRADOS NA ETAPA 2 & EPT-DT & TA \\
\hline 3 & 0 & - & 0 & - \\
\hline
\end{tabular}

Fonte: Elaborado pelos autores.

O Quadro 5 indica os dados obtidos fazendo uso do seguinte critério de pesquisa - Critério 5: C5 = jogo e C6 = educação profissional.

Quadro 5 - Artigos selecionados pelo Critério 5.

\begin{tabular}{|c|c|c|c|c|}
\hline NAE1 & NAE2 & DETALHES DOS ARTIGOS FILTRADOS NA ETAPA 2 & EPT-DT & TA \\
\hline 21 & 0 & - & 0 & - \\
\hline
\end{tabular}

Fonte: Elaborado pelos autores.

O Quadro 6 indica os dados obtidos fazendo uso do seguinte critério de pesquisa - Critério 6: $\mathrm{C5}$ = game e C6 = educação profissional. 
Quadro 6 - Artigos selecionados pelo Critério 6.

\begin{tabular}{|c|c|c|c|c|}
\hline NAE1 & NAE2 & DETALHES DOS ARTIGOS FILTRADOS NA ETAPA 2 & EPT-DT & TA \\
\hline 3 & 0 & - & 0 & - \\
\hline
\end{tabular}

Fonte: Elaborado pelos autores.

O Quadro 7 indica os dados obtidos fazendo uso do seguinte critério de pesquisa - Critério 7: $\mathrm{C} 5$ = serious game e C6 = vocational.

Quadro 7 - Artigos selecionados pelo Critério 7.

\begin{tabular}{|c|c|c|c|c|}
\hline NAE1 & NAE 2 & DETALHES DOS ARTIGOS FILTRADOS NA ETAPA 2 & EPT-DT & TA \\
\hline \multirow{7}{*}{51} & \multirow{7}{*}{7} & $\begin{array}{c}\text { ARTIGO } 08 \text { - Título: Automated Stock Trading - } \\
\text { Developing the Serious Game FSTG to Teach the Topic of } \\
\text { Finite State Machines } \\
\text { Autores: Matthias Christoph Utesch; Andreas Hauer; } \\
\text { Robert Heininger; Helmut Krcmar. }\end{array}$ & 1 & TB \\
\hline & & $\begin{array}{c}\text { ARTIGO } 09 \text { - Título: Bridging behavior science and } \\
\text { gaming theory: Using the Intervention Mapping Protocol } \\
\text { to design a serious game against cyberbullying } \\
\text { Autores: Desmet, Ann; Van Cleemput, Katrien; } \\
\text { Bastiaensens, Sara; Poels, Karolien; Vandebosch, Heidi; } \\
\text { Malliet, Steven; Verloigne, Maïté; Vanwolleghem, Griet; } \\
\text { Mertens, Lieze; Cardon, Greet; De Bourdeaudhuij, Ilse. }\end{array}$ & 0 & DP \\
\hline & & $\begin{array}{c}\text { ARTIGO } 10-(*) \text { Título: Subjective Experience and } \\
\text { Sociability in a Collaborative Serious Game } \\
\text { Autor: Oksanen, Kimmo. }\end{array}$ & 0 & DP \\
\hline & & $\begin{array}{r}\text { ARTIGO } 11 \text { - Título: Food safety and young consumers: } \\
\text { Testing a serious game as a risk communication tool } \\
\text { Autores: Crovato, S.; Pinto, A.; Giardullo, P.; Mascarello, } \\
\text { G.; Neresini, F.; Ravarotto, L. }\end{array}$ & 0 & NATA \\
\hline & & $\begin{array}{c}\text { ARTIGO } 12-\left({ }^{*}\right) \text { Título: Game Mechanics in the Design of } \\
\text { a Collaborative 3D Serious Game } \\
\text { Autores: Oksanen, Kimmo; Hämäläinen, Raija. }\end{array}$ & 0 & NATA \\
\hline & & $\begin{array}{l}\text { ARTIGO } 13 \text { - Título: "It's like you're actually playing as } \\
\text { yourself": Development and preliminary evaluation of } \\
\text { 'Green Acres High', a serious game-based primary } \\
\text { intervention to combat adolescent dating violence } \\
\text { Autores: Bowen, Erica; Walker, Kate; Mawer, Matthew; } \\
\text { Holdsworth, Emma; Sorbring, Emma; Helsing, Bo; Bolin, } \\
\text { Annette; Leen, Eline; Held, Paul; Awouters, Valère; Jans, } \\
\text { Sebastiaan. } \\
\end{array}$ & 0 & $A E$ \\
\hline & & $\begin{array}{l}\text { ARTIGO } 14 \text { - Título: Teaching Literacy Skills to French } \\
\text { Minimally Verbal School-Aged Children with Autism } \\
\text { Spectrum Disorders with the Serious Game SEMA-TIC: An } \\
\text { Exploratory Study } \\
\text { Autores: Serret, Sylvie; Hun, Stéphanie; Thümmler, } \\
\text { Susanne; Pierron, Prescillia; Santos, Andreia; Bourgeois, } \\
\text { Jérémy; Askenazy, Florence. }\end{array}$ & 0 & COM \\
\hline
\end{tabular}

Fonte: Elaborado pelos autores.

O Quadro 8 indica os dados obtidos fazendo uso do seguinte critério de pesquisa - Critério 8: C5 = game e C6 = vocational education. 
Quadro 8 - Artigos selecionados pelo Critério 8.

\begin{tabular}{|c|c|c|c|c|}
\hline NAE1 & NAE2 & DETALHES DOS ARTIGOS FILTRADOS NA ETAPA 2 & EPT-DT & TA \\
\hline \multirow{13}{*}{448} & \multirow{13}{*}{26} & $\begin{array}{c}\text { ARTIGO } 15 \text { - Título: The signal game: Missions and } \\
\text { playground to motivate vocational education students } \\
\text { toward periodic signals } \\
\text { Autor: Marzo, Asier. }\end{array}$ & 1 & $\mathrm{AE}$ \\
\hline & & $\begin{array}{c}\text { ARTIGO } 16 \text { - Título: Design Rationale Behind the Serious } \\
\text { Self-Regulation Game Intervention "Balance It": } \\
\text { Overweight Prevention Among Secondary Vocational } \\
\text { Education Students in The Netherlands } \\
\text { Autores: Spook, Jorinde E; Paulussen, Theo; Paulissen, } \\
\text { Rosie; Visschedijk, Gillian; Kok, Gerjo; van Empelen, } \\
\text { Pepijn }\end{array}$ & 0 & DP \\
\hline & & $\begin{array}{l}\text { ARTIGO } 17 \text { - Título: The Identification of the Potential of } \\
\text { Game-based Learning in Vocational Education within the } \\
\text { Context of the Project "Play the Learning Game" } \\
\text { Autores: Jutta Pauschenwein; Eva Goldgruber; Anastasia } \\
\text { Sfiri. }\end{array}$ & 0 & DP \\
\hline & & $\begin{array}{l}\text { ARTIGO } 18 \text { - Título: The effectiveness of a math game: } \\
\text { The impact of integrating conceptual clarification as } \\
\text { support } \\
\text { Autores: Vandercruysse, Sylke; Ter Vrugte, Judith; de } \\
\text { Jong, Ton; Wouters, Pieter; van Oostendorp, Herre; } \\
\text { Verschaffel, Lieven; Moeyaert, Mariola; Elen, Jan. }\end{array}$ & 0 & DP \\
\hline & & $\begin{array}{c}\text { ARTIGO } 19 \text { - Título: Can Learning Motivation Predict } \\
\text { Learning Achievement? A Case Study of a Mobile Game- } \\
\text { Based English Learning Approach } \\
\text { Autores: Tsai, Chia - Hui ; Cheng, Ching - Hsue; Yeh, } \\
\text { Duen - Yian; Lin, Shih - Yun. }\end{array}$ & 0 & DP \\
\hline & & $\begin{array}{c}\text { ARTIGO } 20 \text { - Título: Students' Viewpoint of Computer } \\
\text { Game for Training in Indonesian Universities and High } \\
\text { Schools } \\
\text { Autores: Wahyudin, Didin; Hasegawa, Shinobu; } \\
\text { Kamaludin, Apep. }\end{array}$ & 0 & DP \\
\hline & & $\begin{array}{c}\text { ARTIGO } 21 \text { - Título: Content Integration as a Factor in } \\
\text { Math-Game Effectiveness } \\
\text { Autores: Vandercruysse, Sylke; Ter Vrugte, Judith; De } \\
\text { Jong, Ton; Wouters, Pieter; Van Oostendorp, Herre; } \\
\text { Verschaffel, Lieven; Elen, Jan. }\end{array}$ & 0 & DP \\
\hline & & $\begin{array}{c}\text { ARTIGO } 22 \text { - Título: Evaluation of a Serious Self- } \\
\text { Regulation Game Intervention for Overweight-Related } \\
\text { Behaviors ("Balance It"): A Pilot Study } \\
\text { Autores: Spook, Jorinde; Paulussen, Theo; Kok, Gerjo; } \\
\text { Van Empelen, Pepijn. }\end{array}$ & 0 & NATA \\
\hline & & $\begin{array}{c}\text { ARTIGO } 23 \text { - Título: Fostering Creativity through } \\
\text { Educational Video Game Development Projects: A Study } \\
\text { of Contextual and Task Characteristics } \\
\text { Autores: Fabricatore, Carlo; López, Ximena. }\end{array}$ & 1 & NATA \\
\hline & & $\begin{array}{c}\text { ARTIGO } 24 \text { - Título: How competition and heterogeneous } \\
\text { collaboration interact in prevocational game-based } \\
\text { mathematics education } \\
\text { Autores: Ter Vrugte, Judith; de Jong, Ton; } \\
\text { Vandercruysse, Sylke; Wouters, Pieter; van Oostendorp, } \\
\text { Herre ; Elen, Jan. } \\
\end{array}$ & 0 & DP \\
\hline & & $\begin{array}{c}\text { ARTIGO } 25 \text { - Título: A Web-based computer-tailored } \\
\text { game to reduce binge drinking among } 16 \text { to } 18 \text { year old } \\
\text { Dutch adolescents: development and study protocol } \\
\text { Autores: Jander, Astrid; Crutzen, Rik; Mercken, Liesbeth; } \\
\text { de Vries, Hein. }\end{array}$ & 0 & NATA \\
\hline & & $\begin{array}{c}\text { ARTIGO } 26 \text { - Título: Promoting science learning in game- } \\
\text { based learning with question prompts and feedback } \\
\text { Autores: Law, Victor; Chen, Ching-Huei }\end{array}$ & 0 & DP \\
\hline & & $\begin{array}{c}\text { ARTIGO } 27 \text { - Título: Teacher perceptions of the value of } \\
\text { game-based learning in secondary education }\end{array}$ & 0 & DP \\
\hline
\end{tabular}




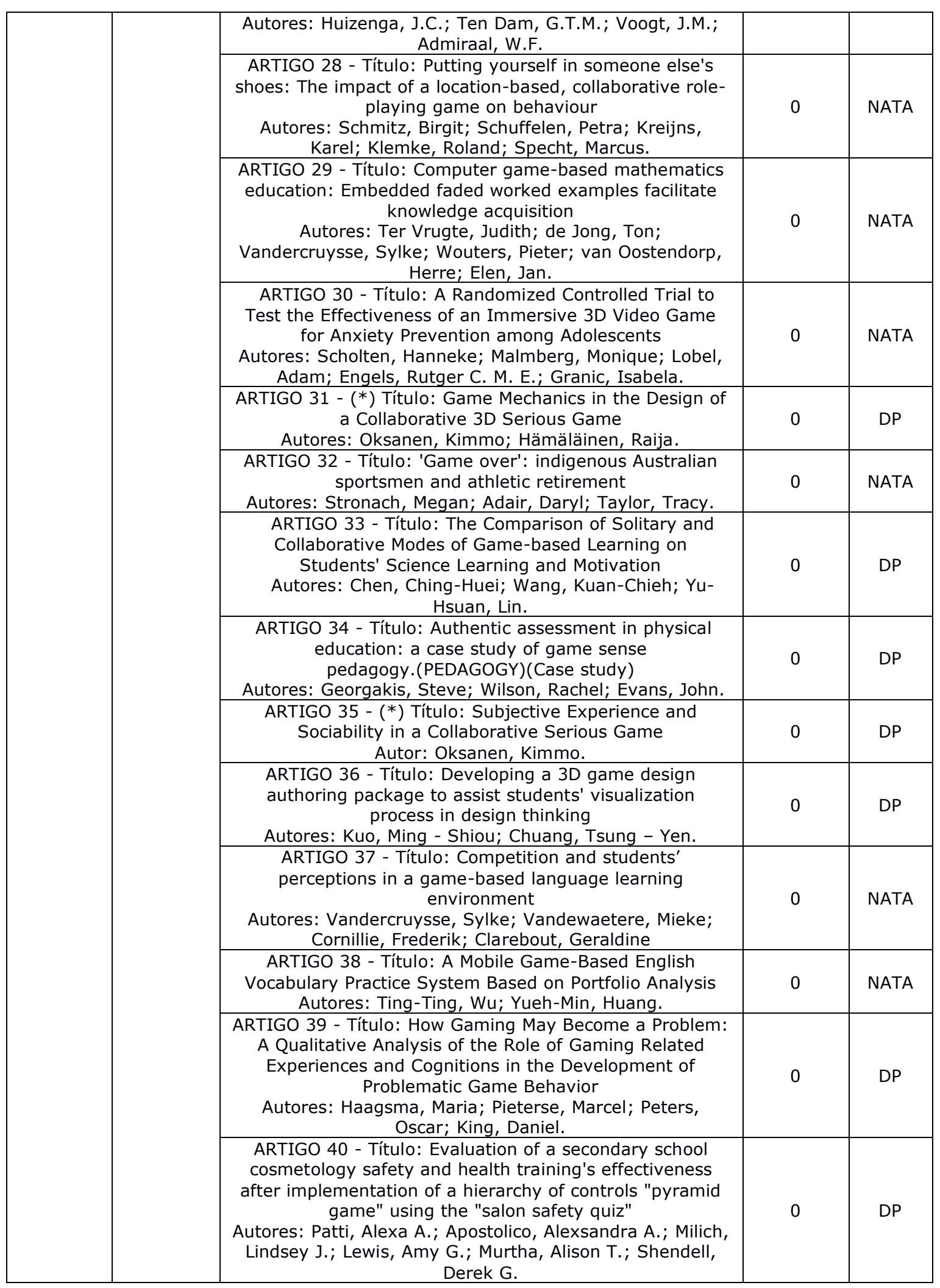

Fonte: Elaborado pelos autores. 
O Quadro 9 indica os dados obtidos fazendo uso do seguinte critério de pesquisa - Critério 9: $\mathrm{C5}$ = digital educational game e C6 = Vazio.

Quadro 9 - Artigos selecionados pelo Critério 9.

\begin{tabular}{|c|c|c|c|c|}
\hline NAE1 & NAE2 & DETALHES DOS ARTIGOS FILTRADOS NA ETAPA 2 & EPT-DT & TA \\
\hline & $\begin{array}{r}\text { ARTIGO 41 - Título: GAMED: digital educational game } \\
\text { development methodology } \\
\text { Autores: Aslan, Serdar; Balci, Osman. }\end{array}$ & DP \\
\cline { 3 - 5 } & \multirow{5}{*}{33} & $\begin{array}{c}\text { ARTIGO 42 - Título: Digital educational game value } \\
\text { hierarchy from a learners' perspective } \\
\text { Autores: Lin, Hong-Wen; Lin, Yu-Ling. } \\
\text { Assuntos: Digital Game-Based Learning; Media in } \\
\text { Education; Simulation Games; Means-End Chains Theory. }\end{array}$ & 0 & DP \\
\cline { 3 - 5 } & $\begin{array}{c}\text { ARTIGO 43 - Título: Pedagogic software evaluation } \\
\text { protocol: analyzing a digital educational game for } \\
\text { portuguese language teaching.(original articles) } \\
\text { Autores: Araujo, Nukacia Meyre Silva; Freitas, Fernanda } \\
\text { Rodrigues Ribeiro. }\end{array}$ & 0 & NATA \\
\hline
\end{tabular}

Fonte: Elaborado pelos autores.

Destaca-se que a pesquisa não encontrou nenhum artigo quando os seguintes critérios foram utilizados: C5 = game educativo e C6 = Vazio; C5 = game educacional e C6 = Vazio e C5 = objeto de aprendizagem e C6 = educação profissional.

Em uma análise dos quadros apresentados, percebe-se que o Número de Artigos Encontrados na Etapa 1 (NAE1), resultou num total de 571 títulos de trabalhos na base de dados de periódicos da Capes. Verificou-se que esse expressivo número ocorreu em razão de que, se a palavra-chave fosse encontrada em qualquer parte do texto, a obra seria filtrada, mesmo que o tema tratado no artigo não fosse relacionado diretamente à aplicação de jogos digitais na educação. Logo, foi necessário a alteração dos filtros para buscar resultados mais consistentes, o que ocorreu na Etapa 2. O Número de Artigos Encontrados na Etapa 2 (NAE2) totalizou 43 artigos, reduzindo a 41 após desconsiderarmos os dois artigos repetidos.

Cada artigo foi especificado por título e autor, conforme quadros apresentados. Em seguida realizou-se a sistematização dos dados referentes aos 41 artigos, visando a criação de gráficos para facilitar o entendimento dos resultados obtidos. Tais ilustrações serão elencadas no decorrer desse estudo.

O artigo 17 foi desconsiderado da pesquisa, devido ao fato de o artigo não abordar um jogo em específico e sim uma análise de um portal de games. Os artigos 32 e 34 também foram excluídos, pois abordam jogos esportivos (boxe, rúgbi e futebol australiano) e assunto específico da disciplina de educação física, respectivamente. Os artigos 09, 10, 16, 18, 19, 20, $21,24,26,27,36,39,40,41$ e 42 foram desconsiderados da pesquisa, uma vez que sua disponibilização não foi encontrada de forma gratuita na internet.

Assim, os percentuais foram determinados conforme ilustrado no gráfico da Figura 2. Dentro de um número total de 41 artigos, foi encontrado um percentual de $56 \%(n=23)$ de artigos classificados que se enquadraram nos critérios estabelecidos para esse estudo. Conforme critérios de exclusão apresentados neste trabalho na seção sobre metodologia, foram 
desconsiderados 37\% $(n=15)$ dos artigos, em razão de serem pagos e $7 \%(n=3)$ dos artigos, por estarem fora do escopo do estudo.

Figura 2 - Gráfico de porcentagem de artigos considerados ou não nesse estudo.

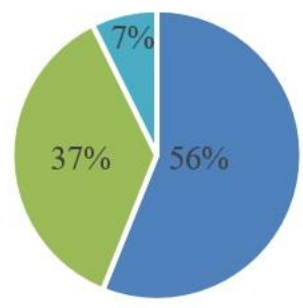

- Artigos Classificados.

- Artigos Pagos Desconsiderados.

- Artigos Fora do Escopo.

Fonte: Elaborada pelos autores.

Quanto ao tipo de teoria de aprendizagem utilizada como referencial teórico nos artigos classificados (um total de 23 artigos), as seguintes análises foram realizadas: 0 autor do artigo 02 não deixa claro a opção pela Teoria de Aprendizagem Humanista, porém o artigo traz uma citação do autor Paulo Freire que é um dos principais autores que seguem a filosofia Humanista. Devido a essa razão, no presente estudo, esse artigo foi considerado como fundamentação Humanista (HUM).

O artigo 03 faz menção ao termo aprendizagem significativa, típica da filosofia Cognitivista e em especial utilizada na teoria do autor David Ausubel. Porém o referido artigo não traz nenhuma referência de autores cognitivistas, logo o artigo foi classificado nesse estudo como Não Apresenta Teoria de Aprendizagem (NATA).

O artigo 04 faz uma análise comparativa entre três jogos no contexto musical. No tópico sobre discussão, o autor desse estudo faz menção a Paulo Freire e seus ensinamentos para a formação autônoma do indivíduo, em um contexto relacionado a jogos digitais. Assim tal trabalho foi classificado como de filosofia humanista (HUM).

Nos demais artigos classificados, conforme critérios estabelecidos nesse trabalho, foi considerada a teoria ou o teórico de aprendizagem explicitamente indicado no texto do artigo. O cenário contendo o número de artigos classificados referente a cada filosofia de aprendizagem, bem como o número de artigos que não apresentaram explicitamente o embasamento teórico está apresentado no gráfico da Figura 3. Destaca-se que em tal gráfico, ainda não há diferenciação entre disciplinas, ou seja, a análise é focada apenas quanto a indicação ou não de teorias de aprendizagem em todos os artigos classificados, independentemente se são sobre JDE para disciplinas propedêuticas ou técnicas.

Figura 3 - Gráfico de número de artigos classificados por filosofia de aprendizagem. Aprendizagem Experiencial (AE); Cognitivismo (COG); Comportamentalismo (COM); Humanismo (HUM); Não Apresenta Teoria de Aprendizagem (NATA); Taxonomia de Bloom (TB). 


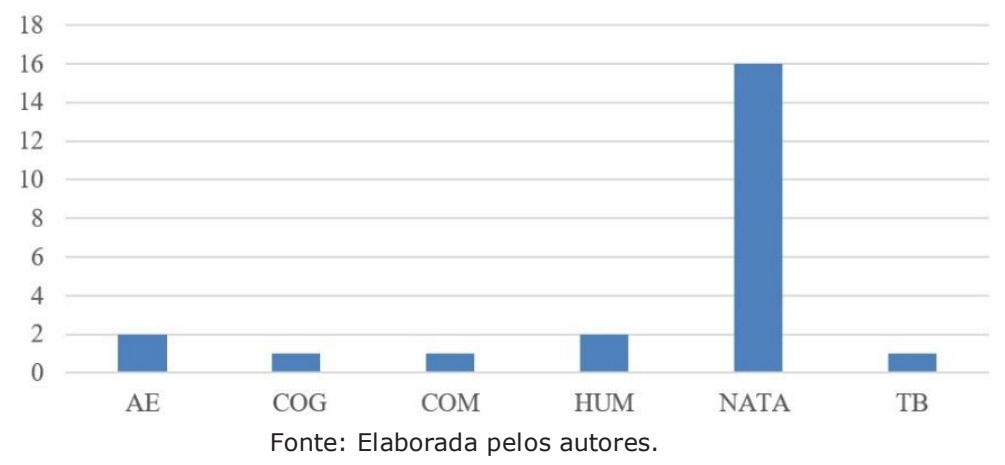

Como última análise, os dados foram sistematizados objetivando verificar a porcentagem de artigos classificados relativos a cada tipo de disciplina, propedêutica ou técnica. As análises descritas a seguir foram realizadas nos artigos que abordam JDE em disciplinas técnicas da EPT.

No artigo 01, o autor não deixa explícito em que modalidade de ensino (disciplina técnica ou propedêutica) ele pode ser aplicado. Como o assunto tratado pelo jogo refere-se a métodos numéricos, que no entendimento dos autores deste estudo, aproxima-se mais das disciplinas técnicas do que propedêuticas, sendo assim considerado como JDE de disciplina técnica de EPT.

O artigo 08 trabalha o tema de Máquinas de Estado Finito, que é típico de curso técnico de nível médio ou superior nas áreas de computação e de algumas engenharias. Em razão disso, o artigo foi considerado dentro do escopo de EPT. No artigo 15, seu autor deixa explícito que a modalidade de ensino na qual JDE foi aplicado é a Educação Profissional (Vocational Education). $\mathrm{O}$ assunto tratado pelo jogo refere-se a processamento de sinais elétricos, sendo utilizado por uma turma de um curso de Educação Profissional de Energias Renováveis em uma instituição de ensino pública espanhola.

O artigo 23 trata de estudo de jogos em uma disciplina de design e desenvolvimento de software multimídia em uma instituição de ensino italiana. No presente estudo, fazendo uma analogia com o contexto do ensino brasileiro, considerou-se essa aplicação como disciplina técnica de EPT.

Logo, constatou-se que apenas $17 \%(n=4)$ dos artigos classificados se enquadraram como específicos de disciplinas da EPT. O restante dos artigos filtrados, ou seja, 83\% ( $n=19$ ), são destinados às aplicações em disciplinas propedêuticas. Esses dados estão apresentados no gráfico da Figura 4. 
Figura 3 - Gráfico de porcentagem de artigos classificados por disciplina.

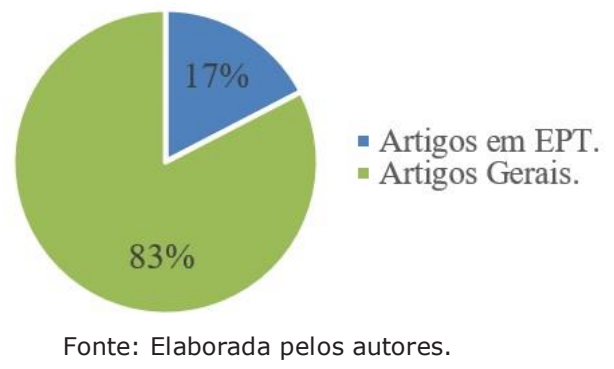

\section{Considerações finais}

O objetivo geral proposto neste estudo foi de verificar qual teoria de aprendizagem é mais citada como fundamento à utilização de JDE na EPT em artigos armazenados na base de periódicos da Capes, publicados de de 2014 a 2018. Os resultados obtidos por meio da análise dos quatro artigos filtrados como sendo destinados à EPT permitem fazer as seguintes considerações: os artigos 01 e 23 não apresentaram explicitamente uma teoria de aprendizagem para fundamentar o uso do JDE, o artigo 8 cita a Taxonomia de Bloom e o artigo 15 faz referência à Teoria de Aprendizagem Experiencial (teoria kolbiana).

É possível destacar que a pequena quantidade de artigos que utilizam JDE na EPT é um forte indicativo de que pouca pesquisa é dedicada a esse tema. Percebe-se, dessa forma, que tal assunto merece ser mais estudado, tendo em vista sua representatividade e importância na formação de alunos de cursos técnicos e tecnólogos no Brasil.

Cabe frisar que, entre os artigos classificados, não houve prevalência de uma teoria de aprendizagem específica para embasar esse tipo de estudo. Tal fato pode sugerir que ainda não há uma tendência de uso de determinada filosofia de aprendizagem para dar suporte teórico pedagógico à utilização de JDE na educação profissional. Aponta-se ainda que, como um dos critérios estabelecidos neste estudo foi o de considerar apenas artigos de acesso público, pode ser que nos artigos pagos haja uma maior representatividade de artigos de JDE em EPT.

O primeiro objetivo específico proposto neste trabalho buscou identificar a terminologia mais usada nos títulos de artigos científicos para fazer referência a JDE. Num total de 23 artigos classificados, considerando inicialmente os trabalhos em língua estrangeira, o termo mais encontrado nos títulos foi "serious game", presente em 07 artigos conforme relação apresentada no Quadro 7. Logo, esse pode ser um indicativo que tal expressão tem sido mais utilizada para nomear um JDE em língua inglesa na literatura científica.

Não houve prevalência de uma determinada nomenclatura na língua portuguesa. Os termos mais citados foram 03 títulos contendo "Jogo digital" (Quadro 1) e 03 usando "Jogo computacional" (Quadro 3). Portanto, levando em conta trabalhos escritos em português, os resultados sugerem uma falta de padronização existente quanto a nomenclatura utilizada para JDE. 
Quanto ao segundo objetivo específico, ou seja, a identificação do número de artigos no portal Capes que tratam especificamente de aplicação de jogos digitais em disciplinas técnicas da EPT, o seguinte resultado foi obtido: foram encontrados apenas 04 artigos no contexto de EPT, num total de 23 considerados como classificados.

Cabe ressaltar que a pesquisa sofreu uma limitação em razão do grande número de artigos que foram selecionados via pesquisa no portal Capes, porém não estão disponibilizados de forma gratuita na internet. Esses representam um percentual de $37 \%(n=15)$ do total de artigos considerados classificados.

Enfim, após análise e descrição dos dados coletados, constatou-se que a hipótese inicial levantada pelos autores foi de fato confirmada, ou seja, existem poucos artigos sobre a utilização de JDE em disciplinas técnicas da EPT. Os resultados apontaram que apenas $17 \%$ ( $n$ $=4$ ) dos artigos classificados, se enquadraram como específicos de disciplinas técnicas. Porém, deve-se enfatizar que tal resultado foi obtido especificamente por meio de uma pesquisa de artigos indexados na base de periódico da Capes, fazendo uso dos filtros de pesquisas definidos pelos autores e, portanto, não pode ser generalizado para contextos mais abrangentes.

Conforme frisado anteriormente, não é possível afirmar taxativamente que há pouca produção de artigos sobre JDE em disciplinas técnicas da EPT pela comunidade científica no contexto geral. Todavia, os resultados alcançados possibilitam vislumbrar um indicativo de que esse tipo de desenvolvimento é ainda tímido. Estudos sobre JDE em disciplinas propedêuticas já são bem difundidos na comunidade científica e grande parte deles sugerem vantagens pedagógicas no uso desse recurso. Nesse sentido, e alargando a área de estudo para além de disciplinas propedêuticas, estudos sobre o desenvolvimento, utilização e análise pedagógica de JDE em disciplinas técnicas da EPT, devidamente fundamentadas por teorias de aprendizagem, podem contribuir na produção de ferramentas educativas que auxiliem o processo de construção do conhecimento dos educandos.

\section{Referências}

ALVES, L. Relações entre os jogos digitais e aprendizagem: delineando percurso. Educação, Formação \& Tecnologias, América do Norte, 1, dez. 2008. Disponível em: https://eft.educom.pt/index.php/eft/article/view/58/38. Acesso em: 25 nov. 2018.

AVEDON, E. M.; SUTTON, B. The study of games. Nova York: John Wiley \& Sons, 1971.

BLOOM, B. S.; KRATHWOHL, D. R.; MASIA, B. Taxonomia de objetivos educacionais: domínio afetivo. Porto Alegre: Globo, 1972.

BRASIL. Ministério da Educação. Portal de Periódicos da Capes, 11 novembro 2000. Disponível em: https://www.periodicos.capes.gov.br/. Acesso em: 25 nov. 2018.

BRASIL. Ministério da Educação. Portal de Periódicos da Capes, 11 novembro 2000a. Disponível em: https://www.periodicos.capes.gov.br/index.php?option=com_pcontent\&view=pcontent\&alia $\mathrm{s}=$ missao-objetivos\&Itemid=126. Acesso em: 25 nov. 2018.

BROUGÈRE, G. Jogo e educação. Porto Alegre: ArtMéd, 2003.

CAILLOIS, R. Os jogos e os homens. Lisboa: Cotovia, 1990. 
FALCÃO, T. P.; FALCÃO, J. T. R.; HAZIN, I. Gamificação na sala de aula: subsídios para a oferta de contexto pedagógico em regime de zona de desenvolvimento proximal. Revista Tecnologias na Educação Ano 8 - Número/Vol.16 - Edição Temática - Congresso Regional sobre Tecnologias na Educação.

FRIGOTTO, G. CIAVATTA, M. Trabalho como princípio educativo. In: SALETE, R.; PEREIRA, I. B.; ALENTEJANO, P.; FRIGOTTO, G. (Org.). Dicionário da educação do campo. Rio de Janeiro: Escola Politécnica Joaquim Venâncio; São Paulo: Expressão Popular, p. 748-759, 2012.

GALVÃO, T. F.; PEREIRA, M. G. Revisões sistemáticas da literatura: passos para sua elaboração. Epidemiol Serv Saude. 2014 jan-mar;23(1):183-4.

HUIZINGA, J. Homo Ludens: o jogo como elemento da cultura. São Paulo: Perspectiva, 28 ed., 1990.

HWANG, G.; WU, P. Advancements and trends in digital game-based learning research: a review of publications in selected journals from 2001 to 2010. British Journal of Educational Technology, v. 43, n. 1, p. E6-E10, 21 jan. 2012.

LÉVY, P. Cibercultura. Rio de Janeiro. 34 ed. 1999.

LIM, C. P. (2008). Spirit of the game: Empowering students as designers in schools? British Journal of Educational Technology, 39(6), 996e1003.

LIMA, J. M. O jogo como recurso pedagógico no contexto educacional. São Paulo: Cultura Acadêmica: Universidade Estadual Paulista, Pró-Reitoria de Graduação, 2008, 157p.

LOURENÇO, C. E. O "estado da arte" da produção de teses e dissertações sobre games - entendidos como forma de comunicação - no banco de dados capes realizadas entre 1987 e 2010. 2012. Universidade de São Paulo, 2012.

MOREIRA, M.A. Teorias de Aprendizagem. 2 ed. ampl. São Paulo: Pedagógica e Universitária, 2017.

MOURA, D. H. Educação Básica e Educação Profissional e Tecnológica: Dualidade Histórica e Perspectivas de Integração. Holos, Natal, v.2, p. 1-27, 2007. Disponível em: http://www2.ifrn.edu.br/ojs/index.php/HOLOS/article/-viewFile/11/1-10. Acesso em: 26 nov. 2018.

PRENSKY, M. (2006). Don't bother me mom - I'm learning. St. Paul: Paragon House.

PRENSKY, M. (2008). Students as designers and creators of educational computer games: Who else? British Journal of Educational Technology, 39(6), 1004 e 1019.

RIBEIRO, R. J. et al. Teorias de Aprendizagem em Jogos Digitais Educacionais: um Panorama Brasileiro. Novas Tecnologias na Educação, CINTED-UFRGS, v. 13 n. 1, julho, 2015. Disponível em: http://seer.ufrgs.br/index.php/renote/article/view/57589. Acesso em: 27 nov. 2018.

VOS, N.; MEIJDEN, H. v. d.; Denessen, E. (2011). Effects of constructing versus playing an educational game on student motivation and deep learning strategy use. Computers \& Education, 56, $127 \mathrm{e} 137$.

VYGOTSKY, L. S. Formação social da mente. 6 ed. São Paulo: Martins Fontes, 1998.

YANG, Y.-T. C.; CHANG, C.-H. (2013). Empowering students through digital games authorship: Enhancing concentration, critical thinking and academic achievement. Computers and Education, 68, 334e344.

Recebido em janeiro de 2019.

Aprovado para publicação em setembro de 2019.

Altair Fábio Silvério Ribeiro

Programa de Pós-Graduação em Educação Profissional e Tecnológica - ProfEPT, Instituto Federal Goiano, Brasil, altair@iftm.edu.br

\section{Roberta Martins Mendonça Gomes}

Programa de Pós-Graduação em Educação Profissional e Tecnológica - ProfEPT, Instituto Federal Goiano, Brasil, roberta.gomes04@gmail.com 


\section{José Roberto Cruz e Silva}

Programa de Pós-Graduação em Educação Profissional e Tecnológica - ProfEPT, Instituto Federal Goiano, Brasil, jrcs86@gmail.com

\section{Jainer Diogo Vieira Matos}

Programa de Pós-Graduação em Educação Profissional e Tecnológica - ProfEPT, Instituto Federal Goiano, Brasil, jainerdiogo@ufg.br

\section{Júlio César Ferreira}

Programa de Pós-Graduação em Educação Profissional e Tecnológica - ProfEPT, Instituto Federal Goiano, Brasil, julio.ferreira@ifgoiano.edu.br

\section{Fernando Barbosa Matos}

Programa de Pós-Graduação em Educação Profissional e Tecnológica - ProfEPT, Instituto Federal Goiano, Brasil, fernando.matos@ifgoiano.edu.br 